\title{
Early Neurodegeneration in the Retina of Type 2 Diabetic Patients
}

\author{
Hille W. van Dijk, ${ }^{1}$ Frank D. Verbraak, ${ }^{1,2}$ Pauline H. B. Kok, ${ }^{1}$ Marilette Stebouwer, \\ Mona K. Garvin, ${ }^{3,4}$ Milan Sonka, ${ }^{3,5}$ J. Hans DeVries, ${ }^{6}$ Reinier O. Schlingemann, ${ }^{1,7}$ and \\ Michael D. Abràmoff $3,4,5$
}

Purpose. The purpose of this study was to determine whether diabetes type 2 causes thinning of retinal layers as a sign of neurodegeneration and to investigate the possible relationship between this thinning and duration of diabetes mellitus, diabetic retinopathy (DR) status, age, sex, and glycemic control (HbA1c).

Methods. Mean layer thickness was calculated for retinal layers following automated segmentation of spectral domain optical coherence tomography images of diabetic patients with no or minimal DR and compared with controls. To determine the relationship between layer thickness and diabetes duration, DR status, age, sex, and $\mathrm{HbA1c}$, a multiple linear regression analysis was used.

Results. In the pericentral area of the macula, the retinal nerve fiber layer (RNFL), ganglion cell layer (GCL), and inner plexiform layer (IPL) were thinner in patients with minimal DR compared to controls (respective difference $1.9 \mu \mathrm{m}, 95 \%$ confidence interval [CI] $0.3-3.5 \mu \mathrm{m} ; 5.2 \mu \mathrm{m}, 95 \%$ CI $1.0-9.3$ $\mu \mathrm{m} ; 4.5 \mu \mathrm{m}, 95 \% \mathrm{CI} 2.2-6.7 \mu \mathrm{m})$. In the peripheral area of the macula, the RNFL and IPL were thinner in patients with minimal DR compared to controls (respective difference 3.2 $\mu \mathrm{m}, 95 \%$ CI 0.1-6.4 $\mu \mathrm{m} ; 3.3 \mu \mathrm{m}, 95 \%$ CI 1.2-5.4 $\mu \mathrm{m}$ ). Multiple linear regression analysis showed DR status to be the only significant explanatory variable $(R=0.31, P=0.03)$ for this retinal thinning.

From the ${ }^{1}$ Department of Ophthalmology, Academic Medical Center, Amsterdam, The Netherlands; ${ }^{2}$ Department of Biomedical Engineering and Physics, Academic Medical Center, Amsterdam, The Netherlands; ${ }^{3}$ Department of Electrical and Computer Engineering, University of Iowa, Iowa City, Iowa; ${ }^{4}$ Veterans Affairs Medical Center, Iowa City, Iowa; ${ }^{5}$ Department of Ophthalmology and Visual Sciences, University of Iowa Hospital and Clinics, Iowa City, Iowa; ${ }^{6}$ Department of Internal Medicine, Academic Medical Center, Amsterdam, The Netherlands; and ${ }^{7}$ Department of Clinical and Molecular Ophthalmogenetics, The Netherlands Institute for Neuroscience, Royal Netherlands Academy of Arts and Sciences (KNAW), Amsterdam, The Netherlands.

Supported by Netherlands Organization for Health Research and Development; National Institutes of Health Grants R01EY017066, EY018853, and EB004640; Research to Prevent Blindness; and The Edward en Marianne Blaauwfonds.

Submitted for publication November 2, 2011; revised March 1, 2012; accepted March 4, 2012.

Disclosure: H.W. van Dijk, None; F.D. Verbraak, None; P.H.B. Kok, None; M. Stehouwer, None; M.K. Garvin, None; M. Sonka, None; J.H. DeVries, None; R.O. Schlingemann, None; M.D. Abràmoff, $\mathrm{P}$

Corresponding author: Hille W. van Dijk, Department of Ophthalmology, Academic Medical Center, PO Box 22660, 1100 DD, Amsterdam, The Netherlands; h.w.vandijk@amc.nl.

Investigative Ophthalmology \& Visual Science, May 2012, Vol. 53, No. 6

Copyright 2012 The Association for Research in Vision and Ophthalmology, Inc.
Conclusions. This study demonstrated thinner inner retinal layers in the macula of type 2 diabetic patients with minimal DR than in controls. These results support the concept that early DR includes a neurodegenerative component. (Invest Ophthalmol Vis Sci. 2012;53:2719-2719) DOI:10.1167/iovs.11-8997

$\mathrm{D}$ iabetic retinopathy (DR) is commonly viewed as a microvascular complication of diabetes mellitus. In addition to vascular changes, structural neurodegenerative changes such as neural apoptosis, loss of ganglion cell bodies, glial reactivity, and reduction in thickness of the inner retinal layers have been described in the earliest stages of DR. This loss of neural tissue agrees with previous functional studies showing neuroretinal deficits in patients with diabetes including electroretinogram abnormalities, loss of dark adaptation and contrast sensitivity, color vision disturbances, and abnormal microperimetry. ${ }^{1-7}$ The introduction of optical coherence tomography (OCT) has allowed imaging and measuring of retinal thickness (RT) with high accuracy, and several groups have been able to show that total RT is decreased in diabetic patients with no or minimal DR compared to normal controls. ${ }^{8-14}$ The high resolution of spectral domain OCT (SD-OCT) allows measurement of the thickness of all individual retinal layers after automated threedimensional segmentation. ${ }^{15,16}$ The layers that can be identified have been interpreted as follows (from the inner to outer surface): retinal nerve fiber layer (RNFL), ganglion cell layer (GCL), inner plexiform layer (IPL), inner nuclear layer (INL), outer plexiform layer (OPL), outer nuclear layer (ONL) + inner segment photoreceptors (IS), outer segment photoreceptors (OS), and retinal pigment epithelium (RPE). A recent study by this group has shown that the decreased total RT that manifests in type 1 diabetic patients with minimal DR is caused by retinal neuropathy characterized by thinning of the GCL in the pericentral area and RNFL in the peripheral area of the macula. ${ }^{17}$ The difference in disease mechanism and management of patients with type 1 and type 2 diabetes mellitus (DM) may also result in differential development of neuropathy in these patients. ${ }^{18}$ The purpose of the present study was to determine whether type 2 diabetes, like type 1 , causes thinning of individual retinal layers while correcting for age, sex, duration of DM, DR status, and glycosylated serum hemoglobin (HbA1c).

\section{Materials ANd Methods}

\section{Participants}

Patients were recruited from the outpatient clinic of the Department of Ophthalmology at the Academic Medical Center (University Hospital, Amsterdam, The Netherlands). Inclusion criteria were type 2 diabetes 
Table 1. Demographics of Patients with Type 2 Diabetes and No or Minimal Diabetic Retinopathy (DR) and Controls

\begin{tabular}{lccc}
\hline \multicolumn{1}{c}{ Parameters } & $\begin{array}{c}\text { No DR } \\
(\boldsymbol{N}=\mathbf{3 9})\end{array}$ & $\begin{array}{c}\text { Minimal DR } \\
(\boldsymbol{N}=\mathbf{2 5})\end{array}$ & $\begin{array}{c}\text { Controls } \\
(\boldsymbol{N}=\mathbf{5 7})\end{array}$ \\
\hline Age (years) & $56 \pm 9$ & $59 \pm 6$ & $58 \pm 12$ \\
Sex (M:F) & $18: 21$ & $10: 15$ & $23: 34$ \\
Duration DM (years) & $8 \pm 7$ & $16 \pm 8$ & NA \\
HbA1c (\%) & $7.6 \pm 1.0$ & $7.9 \pm 0.8$ & - \\
\hline
\end{tabular}

Values are the mean \pm standard deviation for all subjects in each group. NA, not applicable; -, not performed.

and no or minimal DR as evaluated by a retinal specialist through indirect fundoscopy and slit-lamp stereo biomicroscopy. Minimal DR was defined as microaneurysms only, conforming to stage 2 of the International Clinical Diabetic Retinopathy Disease Severity Scale. ${ }^{19}$ Exclusion criteria were refractive error of more than SE +5 or SE -8 diopters in at least one eye; visual acuity below 20/25; significant media opacity; or a history of glaucoma, uveitis, or retinal disease. Ageand sex-matched subjects free of ocular disease, diabetes, hypertension, or other systemic diseases were recruited as controls from among those who accompanied patients visiting the outpatient clinic. All subjects underwent pupillary dilation and an ophthalmologic examination, including slit-lamp biomicroscopy with a handheld lens (SuperField; Volk Optical, Inc., Mentor, $\mathrm{OH}$ ) and OCT imaging (3D OCT-1000; Topcon Corporation, Tokyo, Japan). Age, sex, duration of diabetes, and HbA1c were obtained from the patient charts. The mean HbA1c was calculated from all available HbA1c measurements in the year preceding the study visit. The study adhered to the tenets of the Declaration of Helsinki. Investigative Review Board approval was obtained at both the Academic Medical Center and the University of Iowa, and all participants gave written informed consent.

\section{Optical Coherence Tomography Imaging and Layer Segmentation}

OCT images of the subjects were obtained with SD-OCT using the 3-D volume scan protocol $\left(6 \times 6 \times 2.2 \mathrm{~mm}^{3}\right)$. Nine intraretinal surfaces were segmented automatically for all subjects using the authors' algorithm, which entails a fully three-dimensional graph search approach. ${ }^{15,16}$ The algorithm had previously shown excellent reproducibility of intraretinal layer thickness. ${ }^{20}$ The identified layers between the intraretinal surfaces were interpreted as follows: A, retinal nerve fiber layer (RNFL); B, ganglion cell layer (GCL); C, inner plexiform layer (IPL); D, inner nuclear layer (INL); E, outer plexiform layer (OPL), F, outer nuclear layer (ONL) + inner segments (photoreceptors) (IS); G, outer segments (photoreceptors) (OS); H, retinal pigment epithelium (RPE). One of the authors (HvD), masked to the demographic characteristics and retinopathy status of each subject, marked two retinal areas centered on the fovea: the pericentral area, with an inner diameter of $1 \mathrm{~mm}$ and an outer diameter of $3 \mathrm{~mm}$; and the peripheral area, with an inner diameter of $3 \mathrm{~mm}$ and outer diameter of $6 \mathrm{~mm}$. The mean thickness of each of the layers in the pericentral and peripheral areas was calculated automatically using Image $1.41 .^{21}$

\section{Statistical Analysis}

Statistical analyses were performed with SPSS 16.0.2 for Windows (SPSS, Chicago, IL). Analysis of variance (ANOVA) was used to assess differences in mean age between diabetic patients with no and minimal DR and controls. Mean HbA1c and duration of diabetes were compared using the unpaired $t$-test between patients with no and minimal DR.

Mean layer thicknesses of diabetic patients with minimal DR, diabetic patients without DR, and controls were compared using ANOVA, followed by a Bonferroni post hoc analysis to correct for multiple comparisons. Correlation analysis between individual retinal layers was performed by calculating the Pearson correlation coefficient. A multiple linear regression model was used to determine the relationship between inner retinal layer thickness and the duration of DM, DR status, age, sex, and HbA1c in the diabetic patients.

\section{Results}

Sixty-four subjects with type 2 DM were included. Thirty-nine patients had no DR, and 25 patients showed minimal DR. Diabetes duration was longer and HbA1c higher in the patients with minimal DR as compared to the patients without DR. Fifty-seven controls were included. There was no significant difference in age and sex among patient groups and controls (see Table 1).

The absolute values and differences in retinal layer thickness $(\mu \mathrm{m})$ between patients with type $2 \mathrm{DM}$ and no or minimal DR as compared to normal controls in the pericentral and peripheral area of the macula are given in Tables 2 and 3. In the pericentral area of the macula, the RNFL, GCL, and IPL were thinner in patients with minimal DR compared to controls (respective difference RNFL: $1.9 \mu \mathrm{m}, 95 \%$ CI $0.3-3.5$ $\mu \mathrm{m}$; GCL: $5.2 \mu \mathrm{m}, 95 \%$ CI 1.0-9.3 $\mu \mathrm{m}$; IPL: $4.5 \mu \mathrm{m}, 95 \%$ CI $2.2-$ $6.7 \mu \mathrm{m})$. In the peripheral area of the macula, the RNFL and IPL were thinner in patients with minimal DR compared to controls (respective difference RNFL: $3.2 \mu \mathrm{m}$, 95\% CI 0.1-6.4 $\mu \mathrm{m}$; IPL: $3.3 \mu \mathrm{m}, 95 \%$ CI 1.2-5.4 $\mu \mathrm{m}$ ). The other retinal layers did not show a significant difference in layer thickness. Patients with diabetes but no DR showed no significant difference in any layer thickness in both areas compared to normal controls.

A multivariable regression analysis including diabetes duration, DR status, age, sex, and HbA1c showed that DR

TABLE 2. Mean Layer Thickness Measurements $(\mu \mathrm{m})$ of the Individual Intraretinal Layers in the Pericentral Area of the Macula in Patients with Type 2 Diabetes with No or Minimal DR Compared to Controls

\begin{tabular}{|c|c|c|c|c|c|c|c|}
\hline Parameters & $\begin{array}{c}\text { No DR } \\
(N=39)\end{array}$ & $\begin{array}{c}\text { Mean } \\
\text { Difference }\end{array}$ & $\begin{array}{l}95 \% \text { CI of the } \\
\text { Difference }\end{array}$ & $\begin{array}{l}\text { Minimal DR } \\
(N=25)\end{array}$ & $\begin{array}{c}\text { Mean } \\
\text { Difference }\end{array}$ & $\begin{array}{l}95 \% \text { CI of the } \\
\text { Difference }\end{array}$ & $\begin{array}{l}\text { Controls } \\
(N=57)\end{array}$ \\
\hline RNFL & $22.3 \pm 2.3$ & 0.9 & $-0.5-2.3$ & $21.3 \pm 3.0$ & 1.9 & $0.3-3.5$ & $23.2 \pm 3.0$ \\
\hline GCL & $46.9 \pm 6.4$ & 2.1 & $-1.6-5.7$ & $43.8 \pm 7.8$ & 5.2 & $1.0-9.3$ & $49.0 \pm 7.5$ \\
\hline IPL & $38.3 \pm 3.9$ & 1.5 & $-0.5-3.5$ & $35.3 \pm 3.3$ & 4.5 & $2.2-6.7$ & $39.8 \pm 4.2$ \\
\hline INL & $38.2 \pm 3.3$ & 1.4 & $-3.4-3.2$ & $38.6 \pm 3.6$ & 1.0 & $-1.0-3.1$ & $39.6 \pm 3.8$ \\
\hline OPL & $28.3 \pm 2.9$ & 0.7 & $-1.0-2.3$ & $28.1 \pm 3.0$ & 0.9 & $-1.0-2.7$ & $29.0 \pm 3.5$ \\
\hline $\mathrm{ONL}+\mathrm{IS}$ & $90.6 \pm 9.3$ & 3.1 & $-1.3-7.5$ & $89.3 \pm 9.0$ & 4.4 & $-0.7-9.4$ & $93.7 \pm 8.2$ \\
\hline OS & $26.3 \pm 2.2$ & 0.8 & $-0.3-1.9$ & $26.9 \pm 2.4$ & 0.2 & $-1.0-1.5$ & $27.1 \pm 2.1$ \\
\hline RPE & $39.3 \pm 2.2$ & -0.1 & $-1.2-1.1$ & $39.3 \pm 2.3$ & -0.1 & $-1.4-1.3$ & $39.2 \pm 2.4$ \\
\hline
\end{tabular}

Values are the mean \pm standard deviation for all subjects in each group. The bold values indicate a statistically significant difference between patients and controls. 
TABLE 3. Mean Layer Thickness Measurements $(\mu \mathrm{m})$ of the Individual Intraretinal Layers in the Peripheral Area of the Macula in Patients with Type 2 Diabetes with No or Minimal DR Compared to Controls

\begin{tabular}{|c|c|c|c|c|c|c|c|}
\hline Parameters & $\begin{array}{c}\text { No DR } \\
(N=39)\end{array}$ & $\begin{array}{c}\text { Mean } \\
\text { Difference }\end{array}$ & $\begin{array}{l}\text { 95\% CI of the } \\
\text { Difference }\end{array}$ & $\begin{array}{l}\text { Minimal DR } \\
(N=25)\end{array}$ & $\begin{array}{c}\text { Mean } \\
\text { Difference }\end{array}$ & $\begin{array}{l}\text { 95\% CI of the } \\
\text { Difference }\end{array}$ & $\begin{array}{l}\text { Controls } \\
(N=57)\end{array}$ \\
\hline RNFL & $34.4 \pm 4.2$ & 2.2 & $-0.6-5.0$ & $33.4 \pm 5.8$ & 3.2 & $0.1-6.4$ & $36.6 \pm 5.6$ \\
\hline GCL & $28.9 \pm 2.7$ & -0.3 & $-2.3-1.7$ & $27.0 \pm 4.8$ & 1.6 & $-0.5-3.9$ & $28.6 \pm 3.7$ \\
\hline IPL & $34.4 \pm 3.5$ & 0.2 & $-1.7-2.0$ & $31.3 \pm 3.2$ & 3.3 & $1.2-5.4$ & $34.6 \pm 3.6$ \\
\hline INL & $29.7 \pm 1.9$ & 0.4 & $-1.0-1.8$ & $28.7 \pm 2.5$ & 1.4 & $-0.1-3.0$ & $30.1 \pm 2.9$ \\
\hline OPL & $25.0 \pm 1.5$ & 0.4 & $-0.6-1.4$ & $24.4 \pm 2.0$ & 1.0 & $-0.1-2.2$ & $25.4 \pm 2.1$ \\
\hline $\mathrm{ONL}+\mathrm{IS}$ & $74.6 \pm 7.1$ & 1.3 & $-2.2-4.8$ & $73.2 \pm 5.8$ & 2.7 & $-1.1-6.6$ & $75.9 \pm 6.2$ \\
\hline Os & $23.5 \pm 1.9$ & 0.3 & $-0.7-1.1$ & $23.7 \pm 2.2$ & 0.1 & $-1.1-1.3$ & $23.8 \pm 2.0$ \\
\hline RPE & $39.3 \pm 1.7$ & 0.0 & $-1.0-1.0$ & $39.5 \pm 2.0$ & -0.2 & $-1.4-0.9$ & $39.3 \pm 1.9$ \\
\hline
\end{tabular}

Values are the mean \pm standard deviation for all subjects in each group. The bold values indicate a statistically significant difference between patients and controls.

status was the only significant explanatory variable $(R=0.31$, $P=0.03$ ) for the thinning of the inner retinal layers (RNFL, GCL, IPL) in both the pericentral and the peripheral area of the macula.

\section{Discussion}

The results of this study demonstrated that the inner retinal layers-RNFL, GCL, and IPL-in the macula were thinner in patients with minimal DR compared to controls in an unselected population of patients with type 2 diabetes. In multiple linear regression analysis, DR status-that is, the presence of minimal DR-was the only significant explanatory variable for this retinal thinning, with age, sex, duration of DM, and HbA1c corrected for.

A previous study using identical methods, showed selective loss of thickness of the GCL in the pericentral area and corresponding loss of RNFL thickness in the peripheral area of the macula in type 1 diabetic patients with minimal DR compared to normal controls. ${ }^{17}$ The changes in retinal thickness were comparable in type 1 and 2 diabetes (Figs. 1, 2). This finding is not self-evident, as there are obvious pathophysiological differences between these two types of diabetes. Type 1 diabetic patients have lower plasma insulin concentrations compared to hyperinsulinemic type 2 patients. In the retina, insulin action stimulates neuronal development, differentiation, growth, and survival. Retinal apoptosis can be reversed by systemic insulin therapy, and insulin provides trophic support for retinal neurons..$^{22-24}$

In the previous study with type 1 diabetic patients, there was a significant linear correlation $(R=0.53, P$ value $<0.01)$ between GCL thickness and the duration of diabetes. ${ }^{17}$ In patients with type $2 \mathrm{DM}$, glucose metabolism can be disturbed years before the diagnosis is made. The duration of the disease process is therefore uncertain in patients with type 2 diabetes, and the assessment of a possible correlation between thinning of the inner retinal layers and duration of disease is therefore less precise.

The present study had some limitations. The grading of the severity of DR was done by a single reader through ophthalmologic examination, including indirect fundoscopy and slit-lamp stereo biomicroscopy, instead of the gold standard, namely, seven-field stereoscopic fundus photography assessment by independent trained graders. ${ }^{25}$ However, the patients certainly did not have advanced retinopathy, indicating that the results do apply to the earliest stage of DR.

Type 2 diabetes is often subclinical. It cannot be excluded that some of the controls also had diabetes type 2 , because the HbA1c of the controls was not known. The presence of

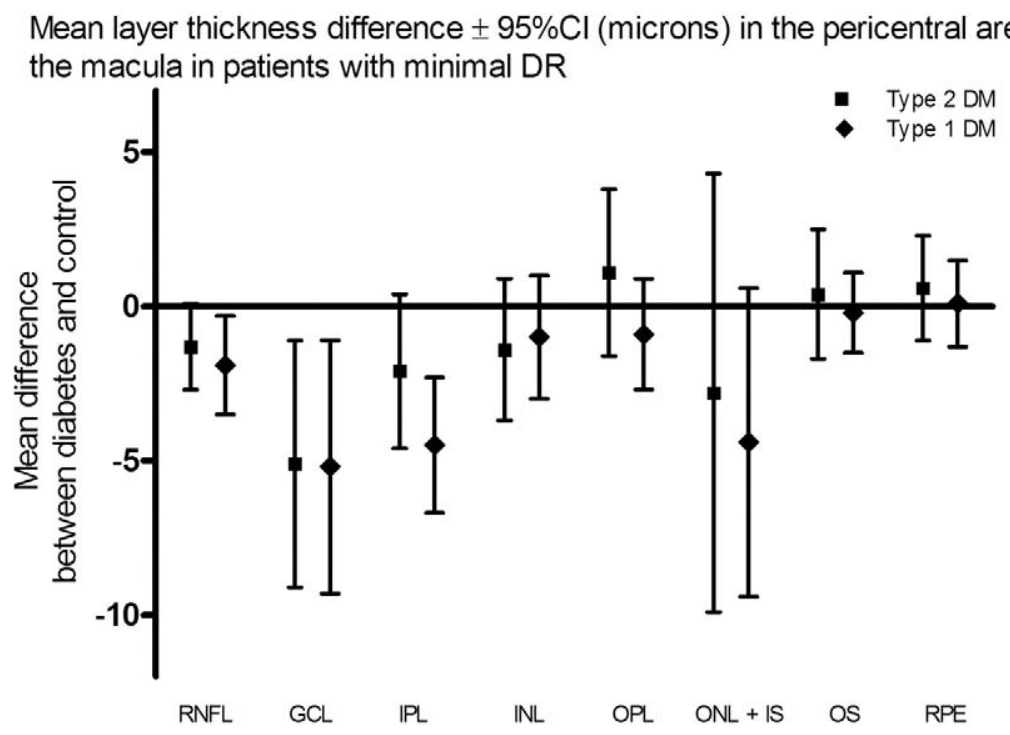

FIGURE 1. Mean layer thickness difference $\pm 95 \% \mathrm{CI}$ (microns) in the pericentral area of the macula in patients with minimal DR. DR, diabetic retinopathy; CI, confidence interval; RNFL, retinal nerve fiber layer; GCL, ganglion cell layer; IPL, inner plexiform layer; INL, inner nuclear layer; OPL, outer plexiform layer; ONL, outer nuclear layer; IS, inner segments; OS, outer segments; RPE, retinal pigment epithelium. 


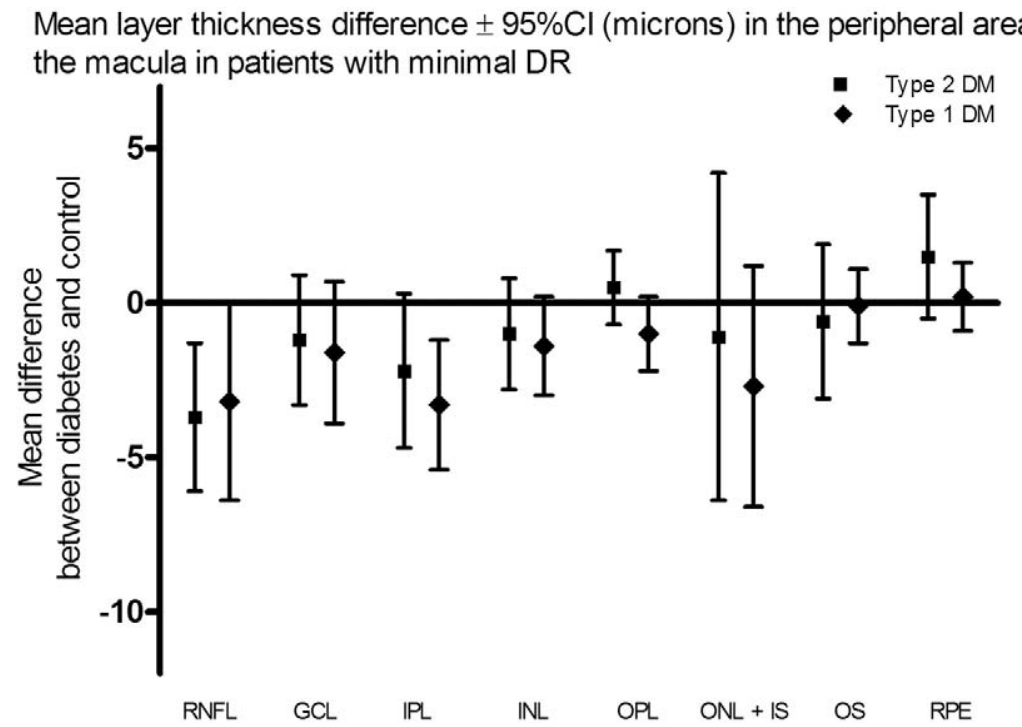

FIGURE 2. Mean layer thickness difference $\pm 95 \% \mathrm{CI}$ (microns) in the peripheral area of the macula in patients with minimal DR. DR, diabetic retinopathy; CI, confidence interval; RNFL, retinal nerve fiber layer; GCL, ganglion cell layer; IPL, inner plexiform layer; INL, inner nuclear layer; OPL, outer plexiform layer; ONL, outer nuclear layer; IS, inner segments; OS, outer segments; RPE, retinal pigment epithelium.

undiagnosed diabetes would most likely lead to underestimation of the difference in retinal layer thickness between patients and controls instead of overestimation.

In a previous study, the present authors' algorithm showed an excellent reproducibility of intraretinal layer thickness. After segmentation of intraretinal layers from repeated OCT scans, the overall mean regional thickness difference was $\mathbf{1 . 1 6}$ $\pm 0.84 \mu \mathrm{m} .{ }^{18}$ This indicates that the resulting mean retinal layer thickness differences in the RNFL, GCL, and IPL between patients and controls in this study exceeded expected differences in intrasubject repeat automated layer thickness measurements. In individual patients, however, repeated retinal layer thickness measurements may not be suitable for detecting small differences in the inner retinal layers. Future studies need to address whether prolonged follow-up measurements of individual patients can show statistically significant thinning over time due to neurodegeneration.

The findings in this study provide further evidence for a neurodegenerative component in early DR. Previous studies have demonstrated neurodegenerative changes including neural apoptosis, loss of ganglion cell bodies, glial reactivity, and reduction in thickness of the inner retinal layers in DR. ${ }^{1,3-14,17}$ These findings of structural neuropathy may explain the neuroretinal functional deficits that are known in patients with diabetes. ${ }^{1-7,11}$ The relationship of this retinal loss to other neural damage in diabetes, such as white matter loss, cognitive decline, peripheral neuropathy, and diabetic autonomic neuropathy, remains to be determined and is the subject of future studies.

In summary, this study provided proof of concept that the inner retinal layers in the pericentral and peripheral area of the macula in type 2 diabetic patients with minimal DR are thinner compared to normal controls. These results support the concept that early DR includes a neurodegenerative component.

\section{References}

1. Antonetti DA, Barber AJ, Bronson SK, et al. Diabetic retinopathy: seeing beyond glucose-induced microvascular disease. Diabetes. 2006;55:2401-2411.
2. Jackson GR, Barber AJ. Visual dysfunction associated with diabetic retinopathy. Curr Diab Rep. 2010;10:380-384.

3. Barber AJ. A new view of diabetic retinopathy: a neurodegenerative disease of the eye. Prog Neuropsychopharmacol Biol Psychiatry. 2003;27:283-290.

4. Barber AJ, Gardner TW, Abcouwer SF. The significance of vascular and neural apoptosis to the pathology of diabetic retinopathy. Invest Ophthalmol Vis Sci. 2011;52:11561163

5. Gardner TW, Abcouwer SF, Barber AJ, Jackson GR. An integrated approach to diabetic retinopathy research. Arch Ophthalmol. 2011;129:230-235.

6. Ghirlanda G, Di Leo MA, Caputo S, Cercone S, Greco AV. From functional to microvascular abnormalities in early diabetic retinopathy. Diabetes Metab Rev. 1997;13:15-35.

7. Van Dijk HW, Verbraak FD, Stehouwer M, et al. Association of visual function and ganglion cell layer thickness in patients with diabetes mellitus type 1 and no or minimal diabetic retinopathy. Vision Res. 2011;51:224-228.

8. Asefzadeh B, Fisch BM, Parenteau CE, Cavallerano AA. Macular thickness and systemic markers for diabetes in individuals with no or mild diabetic retinopathy. Clin Experiment Ophthalmol. 2008;36:455-463.

9. Biallosterski C, van Velthoven ME, Michels RP, Schlingemann RO, DeVries JH, Verbraak FD. Decreased optical coherence tomography-measured pericentral retinal thickness in patients with diabetes mellitus type 1 with minimal diabetic retinopathy. Br J Ophthalmol. 2007;91:1135-1138.

10. Bronson-Castain KW, Bearse MA Jr, Neuville J, et al. Adolescents with type 2 diabetes: early indications of focal retinal neuropathy, retinal thinning, and venular dilation. Retina. 2009;29:618-626.

11. Nilsson M, von Wendt G, Wanger P, Martin L. Early detection of macular changes in patients with diabetes using rarebit fovea test and optical coherence tomography. $\mathrm{Br} J$ Ophthalmol. 2007:91:1596-1598.

12. Oshitari T, Hanawa K, Adachi-Usami E. Changes of macular and RNFL thicknesses measured by stratus OCT in patients with early stage diabetes. Eye. 2009;23:884-889.

13. Van Dijk HW, Kok PH, Garvin M, et al. Selective loss of inner retinal layer thickness in type 1 diabetic patients with minimal 
diabetic retinopathy. Invest Ophthalmol Vis Sci. 2009;50: 3404-3409.

14. Cabrera DD, Somfai GM. Early detection of retinal thickness changes in diabetes using optical coherence tomography. Med Sci Monit. 2010;16:MT15-MT21.

15. Garvin M, Abramoff M, Wu X, Russell S, Burns T, Sonka M. Automated 3-D intraretinal layer segmentation of macular spectral-domain optical coherence tomography images. IEEE Trans Med Imaging. 2009;50:5778-5784.

16. Garvin MK, Abramoff MD, Kardon R, Russell SR, Wu X, Sonka $M$. Intraretinal layer segmentation of macular optical coherence tomography images using optimal 3-D graph search. IEEE Trans Med Imaging. 2008;27:1495-1505.

17. Van Dijk HW, Verbraak FD, Kok PH, et al. Decreased retinal ganglion cell layer thickness in type 1 diabetic patients. Invest Ophthalmol Vis Sci. 2010;51:3660-3665.

18. American Diabetes Association. Diagnosis and classification of diabetes mellitus. Diabetes Care. 2012;35(suppl 1):S64-S71.

19. Wilkinson CP, Ferris FL III, Klein RE, et al. Proposed international clinical diabetic retinopathy and diabetic macular edema disease severity scales. Ophthalmology. 2003;110: 1677-1682.
20. Lee K, Abramoff MD, Sonka M, Garvin M. Automated segmentation of intraretinal layers from spectral domain macular OCT: reproducibility of layer thickness measurements. Proc SPIE Med Imaging. 2011;7965.

21. Abràmoff $M$, Magalhâes $P$, Ram $S$. Image processing with ImageJ. Biophotonics. 2004;11:36-42.

22. Imai H, Singh RS, Fort PE, Gardner TW. Neuroprotection for diabetic retinopathy. Dev Ophthalmol. 2009;44:56-68.

23. Reiter CE, Gardner TW. Functions of insulin and insulin receptor signaling in retina: possible implications for diabetic retinopathy. Prog Retin Eye Res. 2003;22:545-562.

24. Barber AJ, Nakamura M, Wolpert EB, et al. Insulin rescues retinal neurons from apoptosis by a phosphatidylinositol 3kinase/Akt-mediated mechanism that reduces the activation of caspase-3. J Biol Chem. 2001;276:32814-32821.

25. Grading diabetic retinopathy from stereoscopic color fundus photographs-an extension of the modified Airlie House classification. ETDRS report number 10. Early Treatment Diabetic Retinopathy Study Research Group. Ophthalmology. 1991;98:786-806. 